\section{Ion-Coordinating Sensitizer in Solid-State Hybrid Solar Cells**}

\author{
Henry J. Snaith, * Shaik M. Zakeeruddin, \\ Lukas Schmidt-Mende, Cédric Klein, and \\ Michael Grätzel
}

The solid state dye sensitized solar cell is one of the most promising organic based device concepts which demonstrates solar power conversion efficiencies in excess of $4 \% .^{[1,2]}$ The basic strategy of this hybrid system is that light is absorbed in a dye to form an excited electronic state, termed an exciton. Electron transfer takes place from the excited dye molecule to an inorganic semiconductor, where it is transported to and collected at an electrode. Subsequent hole transfer from the

[*] Dr. H. J. Snaith, Dr. S. M. Zakeeruddin, Dr. L. Schmidt Mende,

Dr. C. Klein, Prof. M. Grätzel

Institut de Chimie Physique

École Polytechnique Fédérale de Lausanne

1015 Lausanne (Switzerland)

Fax: (+41) 216934111

E mail: henry.snaith@epfl.ch

[*:*] This work was funded by the MOLYCELL European project. We thank Jacques $E$. Moser for the introduction and use of the transient photovoltage equipment and Michel Schaer and Libero Zuppiroli from the Laboratory of Optoelectronics of Molecular Materials at the EPFL for discussions, assistance with, and use of a low temperature testing rig.

D) Supporting information for this article is available on the WWWW under http://www.angewandte.org or from the author. oxidized dye to a hole transporting organic molecule or polymer takes place, where the hole is transported to and collected at the counter electrode. ${ }^{[3]}$ The fundamental advant age of this system over two component systems ${ }^{[4]}$ is that the electron and hole transporting components are spatially separated by the dye molecules, which hugely suppresses charge recombination and allows efficient charge collection through microns thick material. ${ }^{[8]}$ Furthermore, the efficiency of such devices has been found to dramatically improve with the addition of various additives to the hole transporting material, with the most efficient devices incorporating specific quantities of ionic salts and electrochemical dopants. This "cocktail" of additives has been fine tuned and optimized for the current system. ${ }^{[2]}$ However, the precise function of each component is, as yet, not fully understood. ${ }^{[9]}$ Herein, we report a new charge transfer sensitizer with an ion complex ing moiety formed by oxyethylene side groups. Lithium coordination to the backbone of this dye induces a striking improvement in the photovoltage and performance, with voltages of nearly $900 \mathrm{mV}$ regularly achieved and efficiencies improved from 3.2 to $3.8 \%$ (under $100 \mathrm{~mW} \mathrm{~cm}^{-2}$ simulated air mass (AM) 1.5 solar conditions) for the lithium coordinating sensitizer as compared with a noncoordinating analogue. A staggering record efficiency of $4.6 \%$ is achieved under $10 \mathrm{~mW} \mathrm{~cm}^{-2}$ simulated AM 1.5 illumination. Our supramolec ular approach to the self assembly of functional compo nents ${ }^{[10,11]}$ opens new avenues to control charge separation and recombination at the interface, and gives us a more comprehensive understanding of the mechanisms occurring within this class of solar cells.

The chemical structures of the dyes used for sensitization are shown in Scheme 1. Z907 is an amphiphilic ruthenium
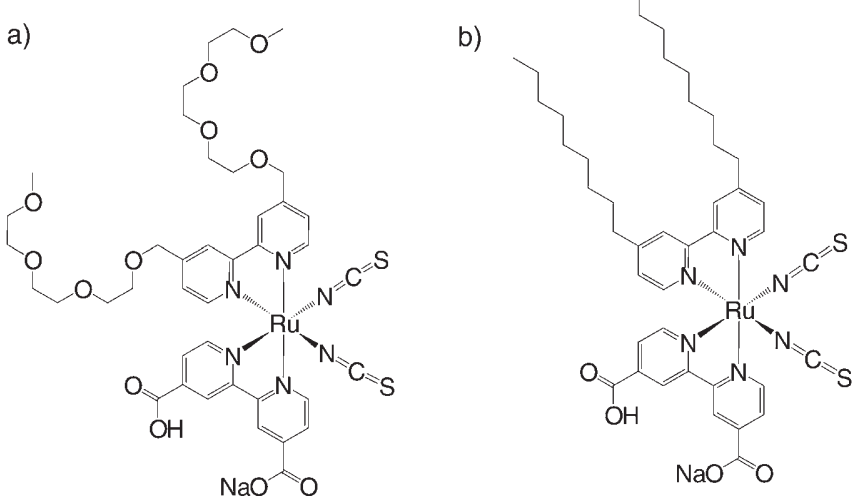

Scheme 1. Structures of the sensitizing dyes used: a) K51 (tetraethy lene oxide side chains) and b) Z907 (nonyl side chains).

complex with one proton and one sodium ion, ${ }^{[12]}$ and K51 is an analogue of Z907, in which the hydrophobic alkyl chains have been replaced with ion coordinating triethyleneglycol methyl ether (TEG) groups. A brief description of the synthetic procedure for this novel sensitizer (K51) is provided in the Supporting Information and will be published in detail elsewhere. ${ }^{[13]}$ The light absorption of the K51 and Z907 dyes is almost identical, both in solution and when adsorbed on a $\mathrm{TiO}_{2}$ nanoporous film. 
Figure 1 a shows the current density voltage $(J V)$ curves in the dark for dye sensitized solar cells. The dark current for the $\mathrm{K} 51$ device at $1.5 \mathrm{~V}$ bias is over ten times larger in the presence of $\mathrm{Li}$ ions than in their absence. There is no color
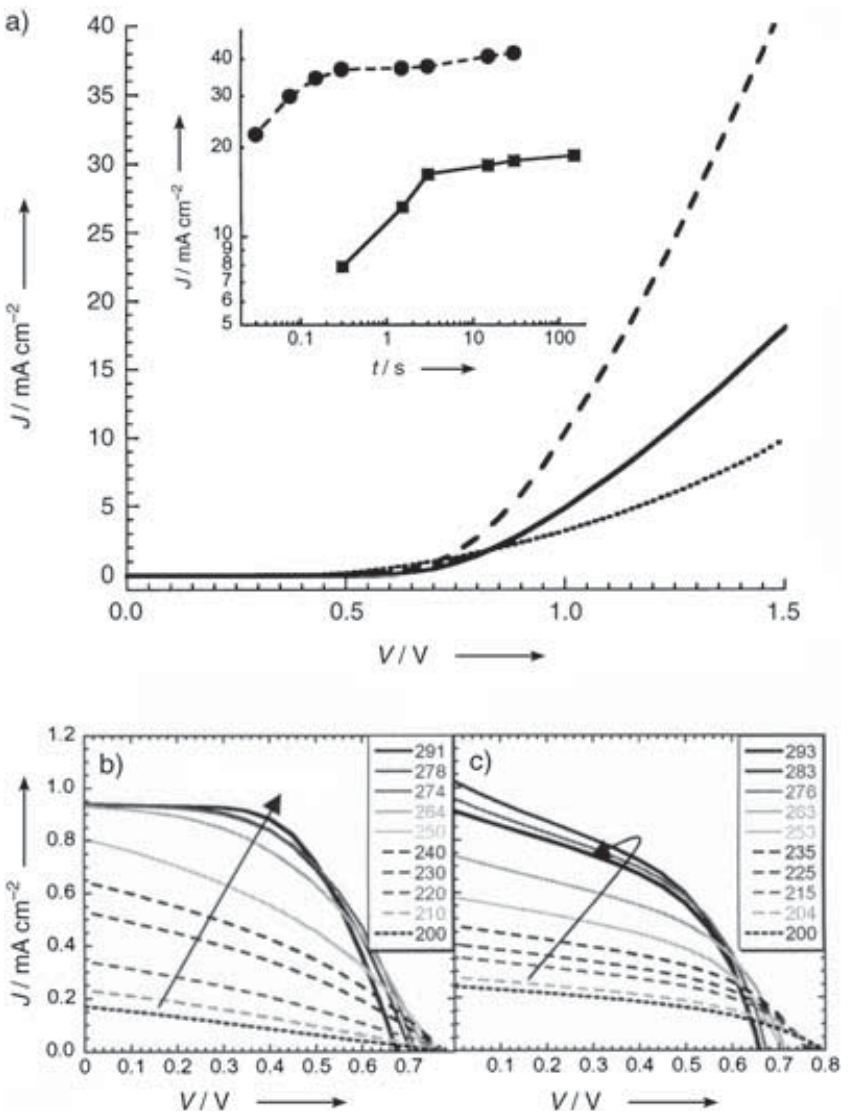

Figure 1. a) Current density $(J)$ measured in the dark versus applied bias ( $V$ ) for a dye sensitized solar cell with $\mathrm{K} 51$ dye in the absence of Li triflate $(\cdots \cdots, \times 10)$, K51 dye with Li triflate ( ), and Z907 dye with Li triflate ( ). Inset: current density at $1.5 \mathrm{~V}$ applied bias versus scan time from 0 to $1.5 \mathrm{~V}$ with the $\mathrm{Z907}$ device $(, \bullet$ ) and the $\mathrm{K} 51$ device $(\quad, \mathbf{a})$. b, c) Current density voltage characteristics for K51 device (b) and Z907 device (c) under illumination from a halo gen lamp $\left(20 \mathrm{mWcm}{ }^{2}\right)$ and at $200300 \mathrm{~K}$ (see legends; the arrows indicate increasing temperatures). These measurements were per formed in the Laboratory of Optoelectronics of Molecular Materials (LOMM), EPFL.

change of the hole transporting material $2,2^{\prime}, 7,7^{\prime}$ tetra $\operatorname{kis}(N, N$ di $p$ methoxyphenylamine) 9,9' spirobifluorene (Spiro) upon the addition of $\mathrm{Li}$ salt, which suggests that no electronic doping takes place. The increased current density could be a consequence of the formation of a dipole at the $\mathrm{Au}$ electrode. ${ }^{[14]}$ However, this would require the bulky triflate anion (negatively charged) to migrate to the gold electrode to improve the hole injection characteristics, which is unlikely. A more reasonable explanation is that the ions in the "electro lyte" can redistribute to balance the space charge, and higher current densities are sustainable as hole transport will no longer be under the "space charge limited" regime. ${ }^{[15]}$ In effect, the addition of the ionic salt dramatically improves the hole mobility in the organic material. Significantly, the current density through the $\mathrm{Li}$ doped $\mathrm{Z} 907$ device is approx imately three times that of the Li doped K51 device, although the initial ionic concentration in both devices is identical. The current density through $\mathrm{Li}$ doped devices varies with the scan rate, with slower scans giving higher currents (this is constant for undoped devices). Notably, this is much more pronounced for the K51 device. The inset to Figure 1 a shows the current density at $1.5 \mathrm{~V}$ versus scan time (from 0 to $1.5 \mathrm{~V}$ ) for the K51 and Z907 Li doped devices. The current for the Z907 device reaches a plateau after approximately $300 \mathrm{~ms}$, whereas the K51 device does not plateau until after $3 \mathrm{~s}$. The increase in current density with time is likely to be a result of the finite time taken for the ions to redistribute and balance the space charge within the device. ${ }^{[15]}$ The only difference between the Z907 and K51 devices is the ion coordinating side chains, so this is direct evidence that a large proportion of the ions are immobilized on the surface of the K51 dye molecules.

Figure $1 \mathrm{~b}$ and $\mathrm{c}$ show the $J V$ characteristics of the K51 and Z907 devices over a range of temperatures under illumina tion. The short circuit current increases with temperature up to around $280 \mathrm{~K}$. The increase in short circuit current over the lower temperature region is consistent with the temperature dependence expected for the hole mobility in an organic hole transporter, ${ }^{[16]}$ with the holes being "frozen" in the device at low temperatures. The fill factor increases with temperature over the entire range studied, but increases most rapidly from around $250 \mathrm{~K}$. An increase in fill factor is consistent with reduced series resistance within the cell $;^{[17]}$ this is likely to be because the ions become more mobile at these higher temperatures. ${ }^{[18]}$ This finding demonstrates that the function of the mobile ions in the hole transporter material is to facilitate a low series resistance, fast charge extraction, and thus a large fill factor.

A striking difference between the two devices is that the short circuit current remains high over the higher range of temperatures for the $\mathrm{K} 51$ device, whereas it drops for the Z907 device with a peak at approximately $280 \mathrm{~K}$. There are two competing temperature dependent factors which influ ence the short circuit current. First, an increased charge mobility will increase the short circuit current by increasing the charge diffusion length ${ }^{[19]}$ and increase the fill factor through reducing the series resistance. ${ }^{[17]}$ Second, the charge recombination rate increases with temperature. ${ }^{[20]}$ An increased charge recombination rate will reduce the short circuit current through reducing the charge diffusion length ${ }^{[19]}$ and reduce the open circuit voltage as it reduces the chemical potential gradient which is sustainable within the device. ${ }^{[21,22]}$ For the Z907 device, it appears that the increasing recombi nation rate becomes dominant at higher temperatures, which results in a drop in short circuit current at these temperatures. However, for the K51 device the increased mobility appears to be dominant over the temperature range studied. The tethering of the $\mathrm{Li}$ ions to the interface is likely to "coulombi cally" retard recombination by screening the electrons in the $\mathrm{TiO}_{2}$ from the holes in the Spiro, thus increasing the activation energy for recombination and retaining a high photocurrent at higher temperatures. This effect appears to be a dominant factor over the increased charge mobility in the Spiro, as the Z907 device, which incorporates the free Li ions, 
exhibits much higher charge transport characteristics. This finding has implications in the use of dual conductive (ion and hole) materials in these solar cells, as the immobilization of ions at the surface seems beneficial. ${ }^{[23,24]}$

Figure $2 \mathrm{a}$ shows the transient decay of the open circuit voltage for various devices. An estimate of the overall charge
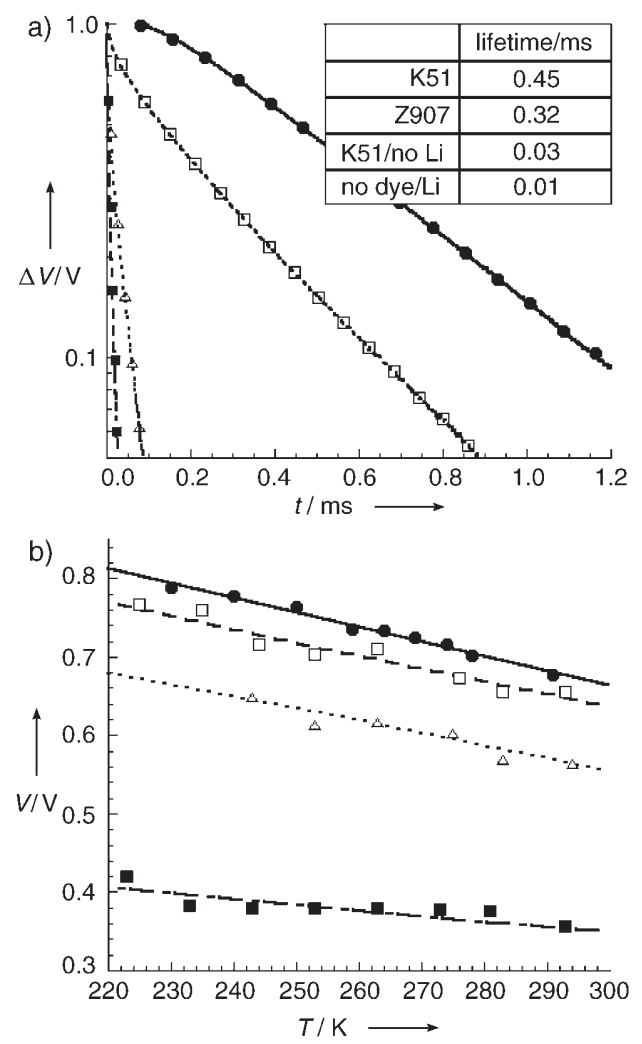

Figure 2. a) Transient open circuit voltage measurements (normalized values) of a Li doped K51 device (•, ) , a Li doped Z907 device ( $\square$,

), a K51 device in the absence of both $\mathrm{Li}$ and $\operatorname{tbp}(\triangle, \cdots .$.$) , and a$ device with only Sb doped Spiro (no dye, Li, or tbp) (घ, -•-•). The inserted table gives the charge lifetimes $\left(\tau_{\text {rec }}\right)$ calculated from the $V_{\text {oc }}$ decay measurements by fitting the decays as $\exp \left(t / \tau_{\text {rec }}\right)$. b) Open cir cuit voltage versus temperature for the same devices as in (a); the symbols are the data points and the lines are linear fits to the data. tbp 4 tert butylpyridine, rec recombination, oc open circuit.

lifetime in these devices can be obtained by simply fitting a monoexponential decay to the curves (see table inset to Figure $2 \mathrm{a}$ ). Charge lifetimes derived in this manner have been shown to give very similar values to those obtained by intensity modulated photovoltage spectroscopy. ${ }^{[2,26]}$ As we might expect, the presence of the dye at the interface increases the charge lifetime (from 10 to $30 \mu \mathrm{s}$ ) as compared to that of a pristine $\mathrm{TiO}_{2}$ film coated with Spiro. Addition of free Li ions (Z907 device) dramatically increases the charge lifetime tenfold to $320 \mu$ s; this effect has been observed by Kruger et al. ${ }^{[9]}$ with transient absorption spectroscopy. Fur thermore, the charge lifetime is increased to $430 \mu$ s by tethering the ions to the dye backbone (K51 device). This result is consistent with the above analysis of the $J V$ data.

Figure $2 \mathrm{~b}$ shows the open circuit voltage as a function of temperature for the same devices. The open circuit voltage drops with increasing temperature over the range studied for all devices. Koster et al. have derived the relationship between open circuit voltage $\left(V_{\text {oc }}\right)$ and absolute temperature $(T)$ for organic PPV:PCBM $(\mathrm{PPV}=\operatorname{poly}(p$ phenyleneviny lene); $\mathrm{PCBM}=[6,6]$ phenyl $\mathrm{C}_{61}$ butyric acid methyl ester) bulk heterojunction photovoltaic devices. ${ }^{[27]}$ We believe the solid state dye sensitized solar cell to be a very similar system, with the only important difference being that there is a dye molecule at the interface between the two components where all excitons are formed. Koster et al. studied the metal insulator metal picture where the material composite is considered to be one intrinsic semiconductor, with the valance band corresponding to the highest occupied molec ular orbital (HOMO) of the hole transporting polymer, and the conduction band corresponding to the lowest unoccupied molecular orbital (LUMO) of the electron withdrawing component (PCBM), with a corresponding energy gap $\left(E_{\text {gap }}\right)$. The relationship is shown in $[\mathrm{Eq}(1)]$ where $\gamma$ is the

$V_{\text {oc }} \quad \frac{E_{\text {gap }}}{q} \frac{k T}{q} \ln \left(\frac{(1 P) \gamma N_{\mathrm{c}}^{2}}{P G}\right)$

Langevin recombination constant, $N_{\mathrm{c}}$ is the effective density of states, $G$ is the generation rate of bound electron hole pairs, $P$ is the dissociation probability of bound electron hole pairs, and $k$ is Boltzmann's constant. For the system studied herein, we consider $G$ to be the rate of generation of electrons in the $\mathrm{TiO}_{2}$ by electron transfer from the photoexcited dye molecule with the hole remaining on the dye, and $P$ to be the probability of hole transfer from the dye molecule to the Spiro.

The linear fit to the data in Figure $2 \mathrm{~b}$ is the relationship we would expect from Equation (1), with the $y$ axis intercept at zero temperature giving $E_{\text {gap. }}$. It is clear that if the active layer in the solid state dye sensitized solar cell can be considered as a single semiconductor material, the exact nature of the interface between the components is critical in determining the electronic structure of such a material. For a $\mathrm{TiO}_{2}$ and Spiro composite $E_{\text {gap }}$ should be approximately $0.6 \mathrm{eV} \cdot^{[1,28]}$ This value is close to that obtained for the device with no dye molecule $(0.56 \mathrm{eV})$, which demonstrates that Equation (1) correctly describes the temperature dependence of the open circuit voltage for this system. However, the derived value of $E_{\text {gap }}$ rises to $1.0 \mathrm{eV}$ when the $\mathrm{TiO}_{2}$ is sensitized with dye molecules, and further to $1.1 \mathrm{eV}$ when $\mathrm{Li}$ is added to the $\mathrm{Z} 907$ device, and to $1.2 \mathrm{eV}$ with surface tethered $\mathrm{Li}$ ions in the $\mathrm{K} 51$ device.

To understand this trend we must consider what the definition of an "energy gap" is in this nanocomposite material. There is no direct transition for an electron in the conduction band of $\mathrm{TiO}_{2}$ to the HOMO level of Spiro, but there is a spatial and energetic barrier in the form of the dye molecule. For this material we should consider the energy gap to be the energy required to move an electron from the $\mathrm{HOMO}$ of the Spiro to the conduction band of $\mathrm{TiO}_{2}$, thus overcoming the barrier presented by the dye molecule. If this is so, the potential barrier is clearly susceptible to changes in local conditions, with the addition of $\mathrm{Li}$ and, more impor tantly, the coordination of $\mathrm{Li}$ ions to the dye molecule 
increasing the barrier height. This results in a widening of the quasi energy gap, with the most important implication being that the fundamental limit of the open circuit voltage $\left(q V_{\text {oc, } \max }=E_{\mathrm{LUMO}}(\right.$ electron transporter $) E_{\mathrm{HOMO}}($ hole trans porter)) is no longer true, as $V_{\text {oc }}$ has an additional contribution (or at least the energy levels can be further offset) because of the exact nature of the interface. This further "energy level offset" is likely to be influenced by the dipole moment which will be present at the interface; the ion coordinating dye molecule will act as a dipole at the surface, and cause a downward shift in the vacuum level of the Spiro with respect to that of the $\mathrm{TiO}_{2} \cdot{ }^{[29}{ }^{31]}$ This shift will force the HOMO level of the Spiro further from the conduction band of the $\mathrm{TiO}_{2}$, thereby increasing the quasi energy gap and thus the open circuit voltage. The introduction of a surface dipole in "flat junction" $\mathrm{TiO}_{2}$ solar cells has previously been shown to increase the open circuit voltage in such devices. ${ }^{[31]}$

Figure 3 shows the current density voltage characteristics for the devices sensitized with the K51 and Z907 dyes, measured under simulated AM 1.5 sunlight at $100 \mathrm{~mW} \mathrm{~cm}^{-2}$.

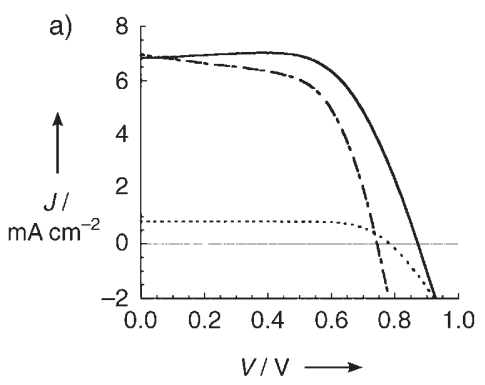

b)

\begin{tabular}{|r|c|c|c|}
\hline & $\begin{array}{c}\mathrm{K} 51 \\
10 \%\end{array}$ & $\begin{array}{c}\mathrm{K} 51 \\
100 \%\end{array}$ & $\begin{array}{c}2907 \\
100 \%\end{array}$ \\
\hline $\begin{array}{c}J_{\mathrm{sc}} \\
{\left[\mathrm{mAcm}^{-2}\right]}\end{array}$ & 0.8 & 6.8 & 6.9 \\
\hline$V_{\mathrm{oc}}[\mathrm{mV}]$ & 785 & 875 & 745 \\
\hline$F F[\%]$ & 75 & 65 & 60 \\
\hline$\eta[\%]$ & 4.6 & 3.8 & 3.2 \\
\hline
\end{tabular}

Figure 3. a) Current density voltage characteristics under simulated AM 1.5 solar conditions $\left(100 \mathrm{mWcm}{ }^{2}\right)$ for a full device containing K51 sensitizing dye ( ) and Z907 sensitizing dye $(-\cdot-\bullet)$. Also shown is the $\mathrm{K} 51$ device illuminated by $10 \mathrm{~mW} \mathrm{~cm}^{2}$ simulated sunlight. b) The standard device performance parameters calculated from the $\mathrm{JV}$ data.

The current densities are similar for the two devices, but both the fill factor and open circuit voltage are improved for the device sensitized with the K51 dye, which leads to an improved efficiency of $3.8 \%$ as opposed to $3.2 \%$ for the Z907 dye. Furthermore, at $10 \mathrm{~mW} \mathrm{~cm}^{-2}$ the efficiency of the K51 device is as high as $4.6 \%$, which demonstrates the potential of this system.

In summary, the presence of mobile ions in the hole transporting material is important as it enables hole transport to take place beyond the space charge limited current regime, dramatically improving the material characteristics. Control of the nature of the interface between the $\mathrm{TiO}_{2}$ and the Spiro, by coordination of $\mathrm{Li}$ ions to the dye backbone, tailors the electronic structure of the composite system to be more favorable for photovoltaic operation. Our findings open new avenues to control charge separation and recombination at the interface of inexpensive bulk junction solar cells, and this work will pave the way for a whole new class of multifunc tional sensitizer molecules.

\section{Experimental Section}

The dye sensitized solar cells were fabricated, and the standard device measurements were performed, by identical methods to those used by Schmidt Mende et al. ${ }^{[2]}$ The additives and concentrations used in a full device were Li triflate $\left(1 \times 10^{20} \mathrm{~cm}^{3}\right.$ in the solid film $), 4$ tert butyl pyridine (tbp; $17 \mu \mathrm{LmL}{ }^{1}$ in chlorobenzene), and antimony salt as the electrochemical dopant $(0.03 \mathrm{~m}$ relative to Spiro). These additives were predissolved in the Spiro solution prior to coating of the sensitized $\mathrm{TiO}_{2}$ film. Tbp was used to help dissolve the Li triflate in the Spiro solution. It has been shown to cause considerable changes to the operation of the device ${ }^{[9]}$ however, here we always used tbp in conjunction with $\mathrm{Li}$ triflate and consider it as one additive for the purposes of analysis. Low temperature current density voltage measurements were performed under high vacuum in the dark and under illumination from an unfiltered halogen lamp with an optical output of $20 \mathrm{~mW} \mathrm{~cm}^{2}$. Transient photovoltage measurements were performed by illuminating the devices with a Nd:YAG laser filtered to give a low intensity $(532 \mathrm{~nm}, 30 \mathrm{~Hz}, 6 \mathrm{~ns}$ pulse width), super imposed upon a large bias, red filtered $(>630 \mathrm{~nm})$, white light illumination from a xenon lamp $\left(\approx 500 \mathrm{~mW} \mathrm{~cm}^{2}\right)$, by a similar method to that described by of O'Regan and Lenzmann. ${ }^{[32]}$

Received: June 10, 2005

Published online: September 14, 2005

Keywords: charge transfer - dyes/pigments · sensitizers . solar cells · supramolecular chemistry

[1] U. Bach, D. Lupo, P. Comte, J. E. Moser, F. Weissortel, J. Salbeck, H. Spreitzer, M. Grätzel, Nature 1998, 395, 583.

[2] L. Schmidt Mende, S. M. Zakeeruddin, M. Grätzel, Appl. Phys. Lett. 2005, 86, 013504.

[3] B. O'Regan, M. Grätzel, Nature 1991, 353, 737.

[4] C. J. Brabec, N. S. Sariciftci, J. C. Hummelen, Adv. Funct. Mater. 2001, $11,15$.

[5] N. C. Greenham, X. G. Peng, A. P. Alivisatos, Phys. Rev. B 1996, 54,17628 .

[6] J. J. M. Halls, C. A. Walsh, N. C. Greenham, E. A. Marseglia, R. H. Friend, S. C. Moratti, A. B. Holmes, Nature 1995, 376, 498.

[7] G. Yu, A. J. Heeger, J. Appl. Phys. 1995, 78, 4510.

[8] L. Schmidt Mende, J. E. Kroeze, J. R. Durrant, M. K. Nazeer uddin, M. Grätzel, Nano Lett. 2005, 5, 1315.

[9] J. Kruger, R. Plass, L. Cevey, M. Piccirelli, M. Grätzel, U. Bach, Appl. Phys. Lett. 2001, 79, 2085.

[10] J. M. Lehn, Proc. Natl. Acad. Sci. USA 2002, 99, 4763.

[11] J. M. Lehn, Science 2002, 295, 2400.

[12] P. Wang, B. Wenger, R. Humphry Baker, J. E. Moser, J. Teuscher, W. Kantlehner, J. Mezger, E. V. Stoyanov, S. M. Zakeeruddin, M. Grätzel, J. Am. Chem. Soc. 2005, 127, 6850.

[13] D. Kuang, C. Klein, H. J. Snaith, R. Humphry Baker, J. E. Moser, S. M. Zakeeruddin, M. Grätzel, 2005, unpublished results.

[14] F. Nuesch, K. Kamaras, L. Zuppiroli, Chem. Phys. Lett. 1998, $283,194$.

[15] J. C. de Mello, N. Tessler, S. C. Graham, R. H. Friend, Phys. Rev. $B$ 1998, 57, 12951.

[16] P. W. M. Blom, M. J. M. de Jong, M. G. van Munster, Phys. Rev. $B$ 1997, 55, R656.

[17] J. Nelson, The Physics of Solar Cells, Imperial College Press, London, 2003.

[18] J. Gao, G. Yu, A. J. Heeger, Appl. Phys. Lett. 1997, 71, 1293.

[19] T. Markvart, L. Castaner, Solar Cells: Materials, Manufacture and Operation, Elsevier, Oxford, 2005.

[20] J. Nelson, S. A. Haque, D. R. Klug, J. R. Durrant, Phys. Rev. B 2001, 6320, 205321. 
[21] B. A. Gregg, M. C. Hanna, J. Appl. Phys. 2003, 93, 3605.

[22] C. M. Ramsdale, J. A. Barker, A. C. Arias, J. D. MacKenzie, R. H. Friend, N. C. Greenham, J. Appl. Phys. 2002, 92, 4266.

[23] S. A. Haque, Y. Tachibana, R. L. Willis, J. E. Moser, M. Grätzel, D. R. Klug, J. R. Durrant, J. Phys. Chem. B 2000, 104, 538.

[24] T. Park, S. A. Haque, R. J. Potter, A. B. Holmes, J. R. Durrant, Chem. Commun. 2003, 2878.

[25] N. Kopidakis, K. D. Benkstein, J. van de Lagemaat, A. J. Frank, J. Phys. Chem. B 2003, 107, 11307.

[26] G. Schlichthorl, S. Y. Huang, J. Sprague, A. J. Frank, J. Phys. Chem. B 1997, 101, 8141.

[27] L. J. A. Koster, V. D. Mihailetchi, R. Ramaker, P. W. M. Blom, Appl. Phys. Lett. 2005, 86, 123509.

[28] M. Grätzel, Nature 2001, 414, 338.

[29] D. M. Alloway, M. Hofmann, D. L. Smith, N. E. Gruhn, A. L. Graham, R. Colorado, V. H. Wysocki, T. R. Lee, P. A. Lee, N. R. Armstrong, J. Phys. Chem. B 2003, 107, 11690.

[30] S. Kera, Y. Yabuuchi, H. Yamane, H. Setoyama, K. K. Okudaira, A. Kahn, N. Ueno, Phys. Rev. B 2004, 70, 085304.

[31] J. Kruger, U. Bach, M. Grätzel, Adv. Mater. 2000, 12, 447.

[32] B. C. O'Regan, F. Lenzmann, J. Phys. Chem. B 2004, 108, 4342. 